\title{
AVALIAÇÃO ENERGÉTICA ECONÔMICA DA CULTURA DO MILHO EM ASSENTAMEN- TO RURAL, IPERÓ-SP ${ }^{1}$ \\ LUÍS CARLOS FERREIRA DE ALMEIDA ${ }^{2}$; OSMAR DE CARVALHO BUENO ${ }^{3} \&$ MAURA SEIKO TSUTSUI ESPERANCINI ${ }^{4}$
}

RESUMO: Tendo como hipótese que maiores inputs energéticos não guardam proporção com outputs econômicos, este trabalho estudou os fluxos energéticos e econômicos da cultura do milho, para os diversos sistemas de produção existentes no Assentamento Ipanema Área I, tendo como ferramental os índices de eficiência Cultural, Energética e Econômica, acrescidos da proposta metodológica dos índices de Eficiência Cultural Econômica e Energética Econômica construídos para cenários probabilísticos. Foram identificados quatro sistemas diferentes: "A", "B", "C" e "D", com dispêndios energéticos, respectivamente,

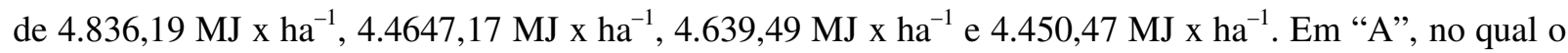
uso de máquinas é mais intensivo, a participação da energia de fonte biológica foi de $23,26 \%$, enquanto os de origem fóssil foi de 76,74\%. O sistema "D" possui a maior Eficiência Cultural, com índice médio de 16,26, enquanto "A" apresentou os menores índices de Eficiência Cultural, com valores médios de 14,83. Para análise da Eficiência Energética, indicativo da dependência de fontes não-renováveis, o maior índice foi o sistema "D", com índice médio de 53,84. Os Índices de Eficiência Econômica, variando entre 1,84 e 1,96 , mostraram que todos os sistemas foram eficientes. O sistema " $D$ ", com índice igual a 8,84, apresentou o mais alto índice de eficiência Cultural Econômica. A análise Energética Econômica para os sistemas "A", "B", "C" e "D", resultou, respectivamente, nos índices de 20,80 , 23,61 , 22,87 e 29,26. Concluiu-se que o uso mais intensivo de energia de fontes não-renováveis (sistema " $\mathrm{A}$ ") não se traduziu necessariamente numa maior eficiência, quando comparado ao "D" (intensivo de mão-de-obra), o que comprova a hipótese inicial do trabalho.

Palavras-chave: Avaliação energética econômica, balanço energético, eficiência energética

\footnotetext{
${ }^{1}$ Parte da Tese de Doutorado do primeiro autor intitulada “Avaliação energética econômica da cultura do milho em assentamento rural, Iperó-SP”.

${ }^{2}$ Aluno do Programa de Pós-graduação em Agronomia - Energia na Agricultura, FCA/UNESP, Botucatu/SP Brasil; e-mail: luis.almeida@uniso.br

${ }^{3}$ Orientador e docente do Departamento de Gestão e Tecnologia Agroindustrial, FCA/UNESP, Botucatu/SP Brasil; e-mail: osmar@fca.unesp.br

${ }^{4}$ Co-orientadora e docente do Departamento de Gestão e Tecnologia Agroindustrial, FCA/UNESP, Botucatu/SP Brasil; e-mail: maura@fca.unesp.br
} 


\section{ENERGY AND ECONOMIC VALUATION OF MAIZE PLANTING IN RURAL SETTLEMENT, IPERÓ / SP}

SUMMARY: This paper, having as a hypothesis that greater energetic inputs don't keep ratio with economical outputs, has tried to study the economical and energetic flows in the maize cultivation to several production systems used in Ipanema Settling Area I, having as analysis instrument the indexes of Cultural, Energetic, Economical efficiency, which were added to the methodological proposal of Cultural Economical and Energetic Economical Efficiency indexes, built for probability settings. Four different systems have been identified: " $A$ ", " $B$ ", " $C$ " and " $D$ ". The energetic expenditure were, respectively, 4,836.19 MJ $x h a^{-1}, 4,4647.17 M J x h a^{-1}, 4,639.49 M J x h a^{-1}$ and 4,450.47 MJ $x h a^{-1}$. In "A", where the use of machines is more intensive, the participation of biological source energy was $23.26 \%$, whereas the ones of fossil origin are $76.74 \%$. The " $D$ " system has the greatest Cultural Efficiency, with average index of 16.26, whereas "A" showed the lowest Cultural Efficiency indexes, with average values of 14.83. For the analysis of the Energetic Efficiency, that indicates the dependence of energy from non renewable sources, the highest index was the "D" system with an average index of 53.84. The Economical Efficiency Indexes, which ranged from 1.84 to 1.96, show that all systems are efficient. The " $D$ " system, with index equal to 8.84, showed the highest index of Economical Cultural efficiency. The Economical Energetic analysis for “ $A$ ", " $B$ ", "C" and " $D$ " systems, has resulted, respectively, in the following indexes: $21.14 ; 23.86$; 22.87 and 29.26. Through the outcome analysis, it was concluded that the more intensive use of energy from nonrenewable sources ("A" system) didn't necessarily mean a higher efficiency when compared to " $D$ " (labor intensive), what comes to prove the paper's initial hypothesis.

Keywords: Economical energetic analysis; energetic balance; energetic efficiency; rural settling; maize.

\section{INTRODUÇÃO}

O processo de desenvolvimento da agricultura, no período posterior à II Guerra, teve como um de seus objetivos buscar ganhos de produtividade mediante o uso de insumos modernos via progressivo aumento de recursos não-renováveis, principalmente aqueles derivados do petróleo, com implicações diretas na sustentabilidade, não só econômica, mas também dos fluxos energéticos envolvidos. Especificamente 
para sistemas de produção na agricultura familiar, essas análises proporcionam uma compreensão do seu grau de sustentabilidade e da sua dependência a inputs energéticos extra propriedade, e o peso que esses inputs têm no processo produtivo torna importante um aprofundamento de como isso ocorre.

Para a análise energética, diversos autores propuseram várias tipologias. Malassis apud Castanho Filho e Chabaribery (1982) enumera três fluxos distintos: "externo", "interno" e "perdidos ou reciclados". Por sua vez a FAO (1976) classifica os recursos energéticos como "renováveis" e "não-renováveis", ao passo que Macedônio e Picchioni (1985) os classificaram dividindo-os em "Energia Primária" e "Energia Secundária". Comitre (1993), classificou os dispêndios energéticos em dois tipos básicos, quais sejam, a "Energia Direta" e "Energia Indireta". A primeira se constitui na soma das energias na forma pelas quais se apresentam, sendo estas divididas em energias "biológicas", "fóssil" e "elétrica". Já a energia indireta correspondia àquela embutida nas máquinas e implementos, equipamentos, insumos e construções, etc.

Carmo et al. (1988) dividiram as entradas energéticas em "Fóssil", "Biológica" e "Industrial". Segundo essa classificação, entende-se como energia biológica, as energias provenientes das fontes humana e animal, bem como as de origem vegetal, por sua vez, tem-se para a energia fóssil as de origem no petróleo e seus subprodutos; finalmente, na energia industrial, incluem-se as máquinas e implementos agrícolas, tanto de tração mecânica quanto de tração animal. Esse tipo de classificação, ainda que com algumas alterações, foi utilizada nos trabalhos de Bueno (2002), Zanini et al. (2003), e Romero (2005), entre outros, e servirá para a estruturação das formas energéticas no presente trabalho.

Freitas et al. (2006), chamam a atenção no sentido que a análise energética, mediante a realização de balanços energéticos, com a utilização de índices de eficiência como instrumentos importantes para a análise de empreendimentos agrícola.

Dessa forma, vários índices têm sido utilizados para essas análises, sendo citados os seguintes índices: "Eficiência Cultural" (PIMENTEL, 1974 apud MELLO, 1986), "Eficiência Ecológica" (HART, 1980) "Produtividade Ecológica" e "Produtividade Cultural", (FLUCK, 1981 apud MELLO, 1986), destacando-se o elaborado por Risoud (2000), denominado de "Eficiência Energética" que, em sua formulação, relaciona as saídas energéticas úteis com o total das energias não-renováveis usadas na produção, permitindo dessa forma, ter uma visão da sustentabilidade da produção frente ao uso de combustíveis fósseis.

Por sua vez, ao realizarmos a análise econômica de um empreendimento, buscamos antes de qualquer outro indicador, analisar sua rentabilidade, podendo os mesmos serem divididos entre métodos tradicionais, fundamentados na teoria financeira (GASLENE et al. 1999) e aqueles não determinísticos, no qual os resultados são apresentados na forma de distribuições de probabilidade (GASLENE et al., 1999; CASAROTTO FILHO; KOPITTKE, 2000). Destaca-se nesse caso o Método de Simulação de Monte Carlo que consiste, segundo Faulin e Juan (2005), numa técnica quantitativa que faz uso da estatística e computadores para simular o comportamento aleatório de sistemas reais, havendo a necessidade, entretanto, do 
conhecimento prévio das distribuições de probabilidade das variáveis envolvidas.

A conjunção da análise energética com a análise econômica é de grande importância ao buscar compreender de que forma se dá a relação de entradas e saídas energéticas quando relacionadas com entradas e saídas econômicas. Permitem identificar não somente quais entradas energéticas têm maior participação no processo produtivo, mas também valorá-las quanto à sua participação no rendimento econômico. Com esse enfoque, temos os trabalhos de Shankar e Tripathi (1997), Pinto (2002) e Esperancini et al. (2005).

Assim, esse trabalho teve como objetivos determinar os itinerários técnicos e/ou sistemas de cultivo da cultura do milho; e para cada um deles obter os índices de eficiência cultural, energética, econômica, cultural econômico e energético econômico, com base em cenários probabilísticos, de tal forma que, para os índices cultural econômico e energético econômico seja possível identificar, independentemente da situação existente, se os sistemas em estudo apresentam eficiência cultural e/ou energética quando comparada à eficiência econômica.

\section{MATERIAL E MÉTODOS}

O trabalho foi desenvolvido junto aos agricultores da Área I do Projeto de Assentamento Fazenda Ipanema no município de Iperó, região sudoeste do Estado de São Paulo, distante aproximadamente $130 \mathrm{~km}$ da Capital, tendo como objeto de estudo a cultura do milho.

\subsection{Composição dos sistemas de plantio}

A composição da amostra seguiu a metodologia na qual se trabalhou com "amostragens dirigidas", de forma que fosse possível analisar a diversidade dos fenômenos mais importantes observados. Não se trabalhou, dessa forma, com amostragens aleatórias, pois essas não asseguravam, justamente por seu caráter intrínseco, a representação e a análise aprofundada da diversidade que os sistemas de produção da atividade milho pudessem assumir (DUFUMIER, 1996). Assim, a amostra foi construída por produtores mais representativos de cada categoria (patamar tecnológico) e de cada tipo de sistema de produção, identificados através de pesquisa junto ao órgão de Assistência Técnica (agrônomos e técnicos agrícolas) e lideranças locais, sendo simplesmente identificados como Assentados. 


\subsection{Coeficientes energéticos}

Após o levantamento de todas as operações, em seus diversos sistemas de cultivos, cada uma delas foi detalhada quanto às suas unidades energéticas, de acordo com os parâmetros elegidos por Bueno (2002), que em seu trabalho indicou as diretrizes quanto à escolha dos coeficientes técnicos para os elementos de cálculo da energia consumida, em J (Joules) e seus múltiplos, de mão-de-obra; sementes; animais de trabalho; combustíveis e outros lubrificantes; máquinas e implementos; e corretivos de solos e fertilizantes químicos.

\subsubsection{Mão-de-obra}

Para a determinação do dispêndio energético nas operações manuais realizadas, foi utilizado o modelo proposto por Carvalho et. al. (1974), adaptado por Bueno (2002). Eles consideram em seus cálculos que o consumo energético está em função do metabolismo basal, o qual leva em conta o peso, a altura, o sexo e a idade dos indivíduos, acrescidos de $8 \%$ para o trabalho da digestão e para a atividade física, ainda que mínima, tendo como base para a apuração dos dispêndios energéticos as expressões propostas por Harris e Benedict apud Mahan et al. (1998), considerando como parâmetros os valores medianos das alturas, massa e idades dos agricultores encontrados em Bueno (2002)

\subsubsection{Produção e sementes}

Para o cálculo do valor energético das sementes utilizadas para plantio, os valores são os correspondentes a 33.229,12 $\mathrm{kJ} \mathrm{x} \mathrm{kg}^{-1}$, conforme estimado por Pimentel et al. (1973). Por sua vez, considerando que a produção é comercializada na forma de MDPS (milho desintegrado com palha e sabugo) foi adotado o valor energético de 13.590,35 kJ x kg-1 (PERALI et al., 2003).

\subsubsection{Máquinas e implementos}

Para a determinação dos coeficientes caloríficos das máquinas e implementos fez-se uso da metodologia proposta por Döering et al. apud Comitre (1993) e Bueno (2002) denominada Depreciação Energética. Tem como parâmetros o peso das máquinas e dos pneus, e depreciá-los durante sua vida útil, res- 
tando ao final somente a energia embutida na matéria prima de seu processo de fabricação. Os coeficientes energéticos a serem utilizados para trator são os definidos por Döering III apud Comitre (1993) de 14.628,6792 $\mathrm{MJ} \mathrm{x} \mathrm{t}^{-1}$ e 13.012,5744 $\mathrm{MJ} \mathrm{x} \mathrm{t}^{-1}$, que também determinou os coeficientes energéticos para implementos e outros equipamentos, correspondendo a 8.628,9948 $\mathrm{MJ} \mathrm{x} \mathrm{t}^{-1}$, para aqueles utilizados até a fase de plantio e de 8.352,6660 $\mathrm{MJ} \mathrm{x} \mathrm{t}^{-1}$ aos utilizados após essa fase. Para pneus o coeficiente energético utilizado foi de $85.829,4 \mathrm{MJ} \mathrm{x} \mathrm{t}^{-1}$.

\subsubsection{Combustíveis, óleos e lubrificantes}

O consumo de óleo Diesel, bem como de óleos lubrificantes, quando disponível, foi determinado

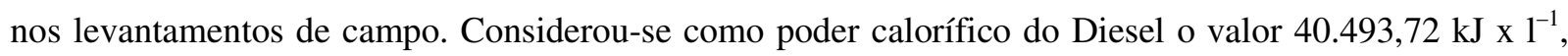
dos óleos lubrificantes 42.370,42 $\mathrm{kJ} \mathrm{x} \mathrm{kg}^{-1}$ e da graxa com 42.705,36 kJ x kg-1 (BRASIL, 2005).

\subsubsection{Fertilizantes químicos}

Os coeficientes calóricos para os fertilizantes utilizados na cultura do milho, acrescidos de 0,5 MJ $\mathrm{x} \mathrm{kg}^{-1}$, correspondente ao transporte marítimo conforme proposto de Leach (1976), são: para N, 62,84 MJ $\mathrm{x} \mathrm{kg}^{-1}$ e para $\mathrm{K}_{2} \mathrm{O} 9,62 \mathrm{MJ} \mathrm{x} \mathrm{kg}^{-1}$, de acordo com Felipe Júnior (1984), por sua vez para $\mathrm{P}_{2} \mathrm{O}_{5}$, os valores estimados são 9,89 MJ x kg${ }^{-1}$ conforme Lockeretz (1980).

\subsubsection{Animais de Tração}

Para o uso de animais de tração, o valor considerado é de $2.400 \mathrm{kcal} \mathrm{x} \mathrm{h}^{-1}$, conforme valores estimados por Odum e Heichel apud Bueno (2002), equivalentes a 10.048,32 $\mathrm{kJ} \mathrm{x} \mathrm{h}^{-1}$.

\subsection{Coeficientes econômicos}

Para o estudo econômico, as despesas diretas foram levantadas junto aos assentados e, quando foi o caso, junto aos próprios prestadores de serviços e insumos, pois esses representam efetivamente o valor executado para a implantação da cultura para a safra 2005/2006. Na falta desses os parâmetros foram obti- 
dos junto ao Instituto de Economia Agrícola (IEA).

\subsection{Análise das eficiências}

A análise das eficiências, para cada um dos sistemas de cultivo, para o período de tempo correspondente, se deu mediante a leitura dos índices de eficiências construídos para a análise econômica, cultural, energética cultural econômica e energética econômica.

A estimação desses índices foi procedida pelo do método de simulação de Monte Carlo, pois permite obter o índice na forma de intervalo de maior probabilidade de ocorrência.

Um dos pressupostos para aplicação do método é o conhecimento a priori das distribuições de probabilidades envolvidas. Assim, especificamente para a estimação dos índices de eficiência econômica, para a variável preço de comercialização, preliminarmente à sua modelagem tem-se a necessidade de retirar o padrão sazonal da série conforme descrito por descritos por Hoffman (1980) e Moretin e Toloi (2004).

Uma vez definidas as distribuições de todas as variáveis envolvidas nas análises econômica, cultural e energética a simulação de Monte Carlo foi realizada conforme proposto por Borges (2003), utilizando-se para tanto o programa de simulação Crystal Ball®.

\subsection{1 Índice de eficiência econômica $\left(I_{\mathrm{EE}}\right)$}

A análise econômica do empreendimento foi realizada por meio do Índice de Eficiência Econômica, conforme equação 1, como se segue:

$I_{E E}=\frac{\left(P_{M} * Q_{M}\right)}{C E}$

$\mathrm{CE}=$ custo total do empreendimento $\mathrm{R} \$ \mathrm{x} \mathrm{ha}^{-1}$;

$\mathrm{P}_{\mathrm{M}}=$ preço do milho $\mathrm{R} \$ \mathrm{x} \mathrm{kg}^{-1}$;

$\mathrm{Q}_{\mathrm{M}}=$ Quantidade de milho, em quilos $\mathrm{xha}^{-1}$; 


\subsubsection{Energética}

\subsubsection{1 Índice de eficiência cultural $\left(\mathrm{I}_{\mathrm{EC}}\right)$}

A obtenção do Índice de eficiência cultural, conforme proposto por Pimentel apud Mello (1986) foi realizada mediante a equação 2 , a seguir:

$$
\mathrm{I}_{\mathrm{EC}}=\frac{\text { "saídas úteis" }(\mathrm{J})}{\text { "entradas" culturais }(\mathrm{J})}
$$

"Entradas" culturais são definidas como a energia contida nos insumos culturais; por sua vez, as "saídas úteis" correspondem aos produtos provenientes de lavouras ou animais (HART, 1980).

\subsubsection{2 Índice de eficiência energética $\left(I_{\mathrm{EEn}}\right)$}

O Índice de Eficiência Energética foi estimado mediante a equação 3, conforme por Risoud (2000), a seguir:

$$
I_{E D M}=\frac{\text { Total energético dos produtos úteis }(\mathrm{J})}{\text { Total das energias não-renowáveis na produção (T) }}
$$

A justificativa da utilização desse índice se prende ao fato da necessidade de aprofundar a análise das energias utilizadas nos diversos sistemas de produção, não só em verificar a existência ou não de eficiência cultural, uma vez que o total energético dos produtos úteis também pode ser entendido como "saídas úteis", conforme Hart (1980), mas também em qualificar o quanto esses sistemas são dependentes da energia proveniente de fontes não-renováveis.

\subsection{3 Índice de eficiência energética econômica}

As relações energéticas econômicas foram avaliadas mediante a análise de dois índices distintos. O primeiro relacionar o Índice de Eficiência Cultural com o Índice de Eficiência Econômica ( $\left.\mathrm{I}_{\mathrm{EE} / \mathrm{EC}}\right)$, conforme equação 4: 


$$
\mathrm{I}_{\mathrm{EC} / \mathrm{EE}}=\frac{I_{E C}}{I_{E E}}
$$

Por sua vez, o segundo foi construído da relação entre os Índices de Eficiência Energética e o Índice de Eficiência Econômica, conforme pode ser visualizado na equação 5:

$$
\mathrm{I}_{\mathrm{EEn} / \mathrm{EE}}=\frac{I_{E E n}}{I_{E E}}
$$

Para a construção desses dois índices, foram utilizados os valores médios (esperança) dos índices de Eficiência Econômica, Cultural e Energética estimados a partir das distribuições de probabilidades, obtidas pela simulação de Monte Carlo.

O quadro 1, a seguir, sintetiza os possíveis resultados de $\mathrm{I}_{\mathrm{EC} / \mathrm{IEE}}$ e $\mathrm{I}_{\mathrm{EEn} / \mathrm{EE}}$ com as suas respectivas interpretações. Nas circunstâncias nas quais os cálculos de $\mathrm{I}_{\mathrm{EC} / \mathrm{IEE}} \mathrm{e} \mathrm{I}_{\mathrm{EEn} / \mathrm{IEE}}$, tenham resultados de sistemas ineficientes energética e/ou culturalmente, utiliza-se um identificador - símbolo de $\left(^{*}\right)$ - o que permite concluir não só quanto às eficiências energéticas e econômicas, mas também se o sistema em si é eficiente do ponto de vista cultural e energético, ou ambos, sendo a identificação realizada na forma como se se segue:

Quadro 1 - Interpretação dos valores dos índices de $\mathrm{I}_{\mathrm{EC} / \mathrm{IEE}} \mathrm{e} \mathrm{I}_{\mathrm{EEn} / \mathrm{IEE}}$.

\begin{tabular}{|c|c|c|c|l|}
\hline Índice & \multicolumn{2}{|c|}{ Valores } & Resultado & \multicolumn{1}{|c|}{ Interpretação } \\
\hline \multirow{5}{*}{$\mathrm{I}_{\mathrm{EC} / \mathrm{EE}}$} & $\mathrm{I}_{\mathrm{EC}} \geq \mathrm{I}_{\mathrm{EE}}$ & $\mathrm{I}_{\mathrm{EC}} \geq 1$ & $\mathrm{I}_{\mathrm{EC} / \mathrm{EE}} \geq 1$ & $\begin{array}{l}\text { Eficiência cultural / econômica, } \\
\text { com Eficiência cultural }\end{array}$ \\
\cline { 2 - 5 } & $\mathrm{I}_{\mathrm{EC}}>\mathrm{I}_{\mathrm{EE}}$ & $\mathrm{I}_{\mathrm{EC}}<1$ & $\mathrm{I}_{\mathrm{EC} / \mathrm{EE}}>1\left(^{*}\right)$ & $\begin{array}{l}\text { Eficiência cultural / econômica } \\
\text { com Ineficiência cultural }\end{array}$ \\
\cline { 2 - 6 } & $\mathrm{I}_{\mathrm{EC}}<\mathrm{I}_{\mathrm{EE}}$ & $\mathrm{I}_{\mathrm{EC}} \geq 1$ & $\mathrm{I}_{\mathrm{EC} / \mathrm{EE}}<1$ & $\begin{array}{l}\text { Ineficiência cultural/econômica } \\
\text { com Eficiência cultural }\end{array}$ \\
\cline { 2 - 6 } & $\mathrm{I}_{\mathrm{EC}}<\mathrm{I}_{\mathrm{EE}}$ & $\mathrm{I}_{\mathrm{EC}}<1$ & $\mathrm{I}_{\mathrm{EC} / \mathrm{EE}}<1(*)$ & $\begin{array}{l}\text { Ineficiência cultural/econômica } \\
\text { com Ineficiência cultural }\end{array}$ \\
\hline \multirow{5}{*}{$\mathrm{I}_{\mathrm{EEn} / \mathrm{EE}}$} & $\mathrm{I}_{\mathrm{EEn}} \geq \mathrm{I}_{\mathrm{EE}}$ & $\mathrm{I}_{\mathrm{EEn}} \geq 1$ & $\mathrm{I}_{\mathrm{EEn} / \mathrm{EE}} \geq 1$ & $\begin{array}{l}\text { Eficiência energética / econômica, } \\
\text { com Eficiência energética }\end{array}$ \\
\cline { 2 - 6 } & $\mathrm{I}_{\mathrm{EEn}}>\mathrm{I}_{\mathrm{EE}}$ & $\mathrm{I}_{\mathrm{EEn}}<1$ & $\mathrm{I}_{\mathrm{EEn} / \mathrm{EE}}>1(*)$ & $\begin{array}{l}\text { Eficiência energética / econômica } \\
\text { com Ineficiência energética }\end{array}$ \\
\cline { 2 - 6 } & $\mathrm{I}_{\mathrm{EEn}}<\mathrm{I}_{\mathrm{EE}}$ & $\mathrm{I}_{\mathrm{EEn}} \geq 1$ & $\mathrm{I}_{\mathrm{EEn} / \mathrm{EE}}<1$ & $\begin{array}{l}\text { Ineficiência energética / econômica } \\
\text { com Eficiência energética }\end{array}$ \\
\cline { 2 - 5 } & $\mathrm{I}_{\mathrm{EEn}}<\mathrm{I}_{\mathrm{EE}}$ & $\mathrm{I}_{\mathrm{EEn}}<1$ & $\mathrm{I}_{\mathrm{EEn} / \mathrm{EE}}<1(*)$ & $\begin{array}{l}\text { Ineficiência energética/econômica } \\
\text { com Ineficiência energética }\end{array}$ \\
\hline
\end{tabular}

Fonte: Dados da pesquisa. 


\section{RESULTADOS E DISCUSSÃO}

Como forma de atingir os objetivos do trabalho de maneira que pudesse permitir uma melhor compreensão dos dados, os resultados foram expressos de forma a apresentar e tipificar os sistemas de cultivo observados e as respectivas matrizes dos coeficientes técnicos e econômicos da produção que compõe os respectivos sistemas de produção.

\subsection{Tipificação dos sistemas de cultivo}

Da análise dos questionários foi possível identificar quatro formas diferentes de como o milho podia ser cultivado no Projeto de Assentamento Fazenda Ipanema - Área I, os quais são descritos (Quadro 2) na forma que segue:

Quadro2 - Síntese das operações realizadas na cultura do milho, Assentamento Ipanema,

Área I, safra 2005/2006.

\begin{tabular}{|c|c|c|c|c|c|}
\hline \multirow[b]{2}{*}{ Sistemas } & \multicolumn{5}{|c|}{ Operações } \\
\hline & $\begin{array}{l}\text { Preparo de } \\
\text { solo }\end{array}$ & Plantio e adubação & $\begin{array}{c}\text { Cultivo e adubação } \\
\text { de cobertura }\end{array}$ & Capina & Colheita \\
\hline "A" & \multirow{4}{*}{ Mecanizado } & \multirow[b]{2}{*}{ Mecanizado } & Mecanizado & - & \multirow{4}{*}{ Manual } \\
\hline "B" & & & $\begin{array}{c}\text { Manual e tração ani- } \\
\text { mal }\end{array}$ & Manual & \\
\hline "C" & & Manual e tracão ani- & Mecanizado & - & \\
\hline "D" & & mal & $\begin{array}{l}\text { Manual e tração ani- } \\
\text { mal }\end{array}$ & Manual & \\
\hline
\end{tabular}

Fonte: Dados da pesquisa

\subsection{Estrutura dos dispêndios energéticos}

A tabela 1, a seguir, apresenta a composição dos dispêndios energéticos, por fontes energética (direta e indireta), por sistema de produção, com seus respectivos valores absolutos e relativos. 
Tabela 1 - Dispêndios energéticos, por sistema de produção em $\mathrm{MJ} \mathrm{x} \mathrm{ha}^{-1}$, assentamento Ipanema, Área I, safra 2005/2006.

\begin{tabular}{l|cc|cc|cc|cc}
\hline \multirow{2}{*}{ Tipo } & \multicolumn{2}{|c|}{ Sistema “A” } & \multicolumn{2}{|c|}{ Sistema “B” } & \multicolumn{2}{|c|}{ Sistema “C” } & \multicolumn{2}{|c}{ Sistema “D” } \\
\cline { 2 - 9 } & MJ & $(\%)$ & MJ & $(\%)$ & MJ & $(\%)$ & MJ & $(\%)$ \\
\hline Energia Direta & $2.451,24$ & 50,69 & $2.266,74$ & 47,78 & $2.259,63$ & 48,70 & $2.075,13$ & 46,63 \\
Energia Indireta & $2.384,95$ & 49,31 & $2.380,43$ & 51,22 & $2.379,86$ & 51,30 & $2.375,34$ & 53,37 \\
& & & & & & & & \\
\hline TOTAL & $4.836,19$ & 100,00 & $4.447,17$ & 100,00 & $4.639,49$ & 100,00 & $4.450,47$ & 100,00 \\
\hline
\end{tabular}

Fonte: Dados da pesquisa

O sistema "A", foi o que apresentou o maior dispêndio energético, com 4.836,199 MJ x ha" ${ }^{-1}$, por sua vez, o sistema " $D$ " foi o que apresentou os menores gastos com 4.450,47 $\mathrm{MJ} \mathrm{x} \mathrm{ha}^{-1}$, já os sistemas "B" e "C" apresentaram gastos energéticos bastante semelhantes, com 4.647,17 MJ x ha ${ }^{-1}$ e 4.639,49 MJ x $\mathrm{ha}^{-1}$, respectivamente.

No sistema "A", no qual o uso de máquinas é mais intensivo, a participação da energia de fonte biológica do tipo direta é de $23,26 \%$, enquanto as de origem fóssil, representada quase que exclusivamente pelo Diesel é de 76,74\%. Na medida em que o uso de máquinas se tornou menos intensivo, sistemas "B", "C" e "D", ocorreu uma significativa redução da participação das energias de fonte fóssil com o conseqüente aumento na participação relativa das energias de fonte biológicas.

A grande participação das energias de fonte biológica é resultado, principalmente, da opção em se realizar a maioria das operações de cultivo de forma manual utilizando, quando necessário, tração animal.

\section{3 Índices de eficiência}

Os índices de eficiências Eficiência Cultural ( $\left.\mathrm{I}_{\mathrm{EC}}\right)$ e Eficiência Energética $\left(\mathrm{I}_{\mathrm{EEn}}\right)$, foram calculados utilizando o método de simulação de Monte Carlo, operacionalizados conforme descrito em material e métodos, e são apresentados na Tabela 2 com os seus valores médios e por percentis: 
Tabela 2 - Resultado dos índices $\mathrm{I}_{\mathrm{EC}}$ e $\mathrm{I}_{\mathrm{EEn}}$, Assentamento Fazenta Ipanema Área I, safra 2005/2006, por sistema de produção.

\begin{tabular}{lcccc|cccc}
\hline \multirow{2}{*}{ Percentil } & \multicolumn{4}{c|}{ Índices de Eficiência Cultural } & \multicolumn{3}{c}{ Índices de Eficiência Energética } \\
\cline { 2 - 9 } & "A" & "B" & "C" & "D" & "A" & "B" & "C" & "D" \\
\hline 25 & 13,39 & 14,14 & 14,01 & 14,88 & 34,61 & 40,96 & 40,79 & 48,77 \\
50 & 14,65 & 15,33 & 15,33 & 16,10 & 37,88 & 44,21 & 44,08 & 53,11 \\
75 & 16,24 & 16,69 & 16,81 & 17,56 & 41,54 & 48,59 & 48,64 & 58,51 \\
\hline Média & 14,83 & 15,48 & 15,46 & 16,26 & 38,27 & 44,86 & 44,82 & 53,84 \\
\hline
\end{tabular}

Fonte: Dados da pesquisa

Do ponto de vista da eficiência cultural, ainda que este apresente resultados mais favoráveis para o sistema "D", quando comparado aos demais, no entanto, pela análise energética se percebe que este sistema é o que apresenta as condições mais favoráveis de sustentabilidade.

Nesse aspecto, pode-se observar que do ponto de vista energético o sistema "A", mais intensivo no uso de máquinas e implementos é o que apresentou o menor índice dentre os quatro sistemas obtidos.

No entanto é de considerar que tanto do ponto de vista cultural, quanto energético que os quatro sistemas são eficientes.

\section{4 Índices de Eficiência Econômica $\left(\mathrm{I}_{\mathrm{EE}}\right)$}

Os resultados da estimação dos Índices de Eficiência Econômica, mostra que todos os sistemas são eficientes economicamente com probabilidade nula de apresentarem eficiência menor que 1 , o que implica em afirmar que essa atividade é viável economicamente para a realidade dos Assentados da Fazenda Ipanema, Área I. Dos quatro sistemas estudados o "C" é o que apresentou os melhores índices de eficiência, com índice esperados entre 1,69 e 2,19 no intervalo interquartil seguido do sistema "B", com valores entre 1,61 e 2,05.

Os sistemas "A", no qual o uso de máquinas e implementos é o mais intenso dentre os quatro, e o "D", intensivo de mão-de-obra, foram os que apresentaram os menores índices de eficiência econômica, com ambos variando praticamente com os mesmos valores no intervalo interquartil, isto é, entre 1,61 e 2,05 . 


\section{5 Índices de Eficiência Cultural Econômica $\left(\mathrm{I}_{\mathrm{EC}} / \mathbf{I}_{\mathrm{EE}}\right)$}

Com base nos valores esperados para os índices cultural e econômico, foi possível calcular o Índice de Eficiência Cultural Econômica para cada um dos sistemas de produção para a cultura do Milho. O sistema que apresentou a melhor relação cultural energética é o sistema "D", com índice igual a 8,84, isto é, para cada unidade de eficiência econômica o sistema responde com quase nove unidades de eficiência cultural.

O sistema com o menor índice de Eficiência Cultural Econômica é "C", e isso se explica em virtude de que, ainda que o mesmo apresente o maior índice econômico, isso não se traduziu em retornos culturais da mesma magnitude. Situação semelhante observa-se nos sistemas "B" e "A".

\section{6 Índices de Eficiência Energética Econômica $\left(\mathrm{I}_{\mathrm{EEn}} / \mathrm{I}_{\mathrm{EE}}\right)$}

Os cálculos dos índices de Eficiência Energética Econômica foram executados com o intuito de verificar qual o nível de resposta os sistemas estudados apresentam em referência às relações energéticas econômicas.

A melhor relação energética econômica observou-se no sistema "D", mais intensivo de mão-deobra, com uso de máquinas e implementos apenas na fase de preparo de solo, que apresentou índice 29,26. Por sua vez, o sistema "A”, com índice 20,80, no qual o uso de equipamentos é o mais intenso dentre os 4 sistemas estudados, é o que apresentou a menor relação energética econômica.

Dessa forma, os quatro sistemas foram eficientes energética, econômica e energética economicamente, apontando para uma melhor relação desses em "D", seguido de "B", com 23,61 e "C"com 22,87, e finalmente " $\mathrm{A}$ ", com todos eles, no curto prazo, sustentáveis economicamente, e no longo prazo mais favorável a "D".

Finalmente, pode-se verificar que o uso mais intensivo de fontes de energias não-renováveis (sistema "A") não se traduziu necessariamente numa maior eficiência econômica desse sistema quando comparado notadamente ao sistema " $D$ " (intensivo em mão-de-obra), o que comprova a hipótese inicial, que altos inputs energéticos não necessariamente se traduzem em maiores retornos econômicos, para as condições de agricultura familiar praticada em Assentamentos de Reforma Agrária. 


\section{CONCLUSÕES}

Foram identificadas quatro formas diferentes pelo qual o milho pode ser cultivado no Projeto de Assentamento Fazenda Ipanema, Área I (sistemas “A", "B", "C" e "D"). No sistema "A", no qual o uso de máquinas é mais intensivo, a participação da energia de fonte biológica de tipo direta foi de $23,26 \%$, enquanto as de origem fóssil, representado quase que exclusivamente pelo óleo Diesel foi de 76,74\%.

Com referência aos índices de $\mathrm{I}_{\mathrm{EC}}$ e $\mathrm{I}_{\mathrm{EEN}}$, os quatros sistemas se mostraram eficientes, com uma nítida vantagem para "D" em oposição ao sistema "A", variando entre um mínimo de 14,83 a 16,26 para $\mathrm{I}_{\mathrm{EC}}$ e entre 38,27 e 53 , para $\mathrm{I}_{\mathrm{EEn}}$.

Uma vez que todos os sistemas apresentaram $\mathrm{I}_{\mathrm{EEn}}$ superior à unidade pode-se concluir que todos estes são eficientes economicamente, podendo-se concluir o mesmo para $\mathrm{I}_{\mathrm{EE}}$, cujos índices também apresentaram valores superiores à unidade.

Para a análise Energética Econômica, foram obtidos os índices 20,80 , 23,61 , 22,87 e 29,26 , respectivamente para os sistemas "A", "B", "C" e "D", indicando serem todos eficientes. A melhor relação Energética Econômica foi observada no sistema "D", seguidos de "B" e "C", que pouco se diferenciaram entre si e pelo sistema "A". Conclui-se dessa forma que os quatro sistemas foram eficientes energética, econômica e energética economicamente, o que indica existir sustentabilidade no curto e longo prazo. Finalmente, pela análise dos valores desses índices que o uso mais intensivo de energia de fontes nãorenováveis, notadamente observados no sistema "A", não se traduziu necessariamente numa maior eficiência econômica desse sistema.

\section{REFERÊNCIAS}

BORGES, C.L.T. Confiabilidade de sistemas de potências. Rio de Janeiro: EE-COPPE/UFRJ, 2003

Disponível em: <http://www.coep.ufrj.br/\%7Ecarmen/CPE754.html>. Acesso em 14 jul. 2006.

BRASIL. Ministério de Minas e Energia. Balanço energético nacional 2004. Brasília: MME, 2005.

Disponível em:

$<$ http://www.mme.gov.br/site/menu/select_main_menu_item.do?channelId=1432\&pageId=4060>. 
Acesso em: 29 set. 2006.

BUENO, O.C. Análise energética e eficiência cultural do milho em assentamento rural, Itaberá/SP. 2002. 146 f. Tese (Doutorado em Agronomia/Energia na Agricultura)-Faculdade de Ciências Agronômicas, Universidade Estadual Paulista, Botucatu, 2002.

CARMO, M.S.; COMITRE, V.; DULLEY, R.D. Balanço energético de sistemas de produção na agricultura alternativa. Agricultura em São Paulo, São Paulo, v. 35, n. 1, p. 87-97, 1988.

CARVALHO, A.; GONÇALVES, G.G.; RIBEIRO, J.J.C. Necessidades energéticas de trabalhadores rurais e agricultores na sub-região víticola de "Torres". Oeiras: Instituto Gulbencvkian de Ciência, Centro de Estudos de Economia Agrária, 1974. 79 p.

CASAROTTO FILHO, N.; KOPITTKE, B.H. Análise de investimentos: matemática financeira, engenharia econômica, tomada de decisão, estratégia empresarial. 9. ed. São Paulo: Atlas, 2000. 458 p.

CASTANHO FILHO, E.P.; CHABARIBERY, D. Perfil energético da agricultura paulista. São Paulo: IEA, Secretaria de Agricultura e Abastecimento, 1982. 55 p. (Relatório de pesquisa, 9/82).

COMITRE, V. Avaliação energética e aspectos econômicos da filière soja na região de Ribeirão Preto - SP. 1993. 132 f. Dissertação (Mestrado em Engenharia Agrícola/Planejamento Agropecuário)Faculdade de Engenharia Agrícola, Universidade Estadual de Campinas, Capinas, 1993.

DUFUMIER, M. Les projets de développement agricole: manuel d'expertise, Paris: CTA-Karthala, 1996. $354 \mathrm{p}$. 
ESPERANCINI, M.S.T. et al. Análise da relação energética e econômica da produção de milho em agricultura familiar no município de Pratânia - SP. In:Congresso Argentino de Ingeniería Rural, 7, 2005, Buenos Aires. Anais... Buenos Aires: Editorial Faculdad de Agronomia, 2005. p. 201-206

FOOD AND AGRICULTURE ORGANIZATION. El estado mundial de la agricultura y la alimentación. Roma, 1976. 158 p.

FAULIN, J.; JUAN, Á.A. Simulación de Monte Carlo com Excel. Técnica Administrativa, Buenos Aires, v. 5, n. 1, jul./sept. 2005. Disponível em:

<http://cyta.com.ar/biblioteca/bddoc/bdlibros/monte_carlo/monte_carlo.htm>. Acesso em: 4 out. 2005.

FELIPE JÚNIOR, G. Considerações sobre a evolução da indústria de fertilizantes nitrogenados. In: SIMPÓSIO SOBRE FERTILIZANTES NA AGRICULTURA BRASILEIRA, 1., 1984, Brasília, DF. Anais... Brasília, DF: EMBRAPA, DEP, 1984. p. 21-71.

FREITAS, S.M. de; OLIVEIRA, M.D.M.; FREDO, C.E. Análise comparativa do balanço energético do milho em diferentes sistemas de produção. In: CONGRESSO DA SOBER, 44, 2006, Fortaleza. Anais... . Campinas: SOBER, 2006. p. 1-13.

GASLENE, Alain; FENTERSEIFER, Jaime E.; LAMB, Roberto. Decisões de investimentos da empresa. São Paulo: Atlas, 1999. 293 p.

HART, R.D. Una metodologia para analizar sistemas agrícolas em terminos energéticos. In: HART, R.D.; JIMÉNZ CH., T.; SERPA, R. Analisis energetico de sistemas agricolas: estudios de casos de producción y procesamiento de caña de azúcar em trapiches, ingenios y distilerías. Turrialba, Costa Rica: CATIE, 1980. p. 3-14. Documento provisional. 
HOFFMAN, R. Estatística para economistas. São Paulo: Pioneira, 1980. 379 p.

LEACH, G. Energy and food production. London: International Institute for Environment and Development, 1976. $192 \mathrm{p}$.

LOCKERETZ, W. Energy inputs for nitrogen, phosphorus and potash fertilizers. In: PIMENTEL, David. (Ed.). Handbook of energy utilization in agriculture. Boca Raton, Florida: CRC Press, 1980. p. 23-26.

MACEDÔNIO, A.C., PICCHIONI, S.A. Metodologia para o cálculo do consumo de energia fóssil no processo de produção agropecuária. Curitiba: DERAL/SEAB, 1985. v. 1, 95 p.

MAHAN, L.K.; ESCOTT-STUMP, S.; KRAUSE, M.V. Krause: alimentos, nutrição e dietoterapia. 9. ed. São Paulo: Roca, 1998. 1179 p.

MELLO, R. Análise energética de agroecossistemas: o caso de Santa Catarina. 1986. 138 f. Dissertação (Mestrado em Engenharia)-Departamento de Engenharia de Produção e Sistemas, Universidade Federal de Santa Catarina, Florianópolis, 1986.

MORETTIN, P.O.; TOLOI, C.M. C. Análise de séries temporais. São Paulo: Edgard Blücher, 2004. $535 \mathrm{p}$.

PERALI, C. et al. Valores nutricionais de alimentos para eqüinos. Revista Brasileira de Zootecnia, Viçosa, v. 32, n. 3, p. 538-545, 2003.

PIMENTEL, D. et al. Food production and the energy crises. Science, Washington, v. 182, p. 443-449, Nov. 1973. 
PINTO, M.S.V. Análise econômica e energética de sistema agroflorestal para implantação na terra indígina Araribá - Município de Avaí, SP. 2002. 136 f. Dissertação (Mestrado em Agronomia/Energia na Agricultura)-Faculdade de Ciências Agronômicas, Universidade Estadual Paulista, Botucatu, 2002.

RISOUD, B. Energy efficiency of various french farming systems: questions to sustainability. INRA, 2000. Disponível em: <http://www.dijon.inra.fr/esr/documents/wp2000_9.pdf>. Acesso em: 16 ago. 2006.

ROMERO, M.G.C. Análise energética e econômica da cultura do algodão em sistemas agrícolas familiares. 2005. 139 f. Dissertação (Mestrado em Agronomia/Energia na Agricultura)-Faculdade de Ciências Agronômicas, Universidade Estadual Paulista, Botucatu, 2005.

SHANKAR, T.U.; TRIPATHI R. S. Evaluating second year cropping on jhum fallows im Mizoran, northeastern Índia: Energy and economics efficiencies. Journal of Biosciences, Bangalore, v. 22, n. 5, p. 605613, dec. 1997.

ZANINI, A. et al. Análise do consumo de energia na produção de silagem de milho em plantio direto.

Acta Scientiarum. Animal Sciences, Maringá, v. 25, n. 2, p. 249-253, 2003. 